\title{
Cardiovascular Correlates of Motor Vehicle Accident Related Posttraumatic Stress Disorder and its Successful Treatment
}

\author{
Sirko Rabe, ${ }^{1,5}$ Denise Dörfel, ${ }^{1}$ Tanja Zöllner, ${ }^{2}$ Andreas Maercker, ${ }^{3}$ and Anke Karl ${ }^{1,4}$ \\ Published online: 9 November 2006
}

\begin{abstract}
Persons with posttraumatic stress disorder (PTSD) have been shown to display elevated baseline cardiovascular activity and a heightened physiological reactivity to trauma-related stimuli. Study 1 examined differences in baseline heart rate $(H R)$ and HR reactivity in 68 survivors of motor vehicle accidents (MVAs) and healthy controls without MVA. MVA survivors with PTSD $(n=26)$, subsyndromal PTSD $(n=22)$, traumatized controls without PTSD (non-PTSD with MVA, $n=20)$ and healthy controls without MVA $(H C, n=27)$ underwent measurement of HR during baseline and exposure to a neutral, positive, negative, and trauma-related picture. PTSD patients showed elevated baseline HR and increased HR reactivity only during exposure to the trauma-related picture. Study 2 investigated whether the elevated physiological responses observed in Study 1 normalized after cognitive behavioral therapy $(C B T)$. We conducted a randomized, controlled treatment trial comparing $C B T(n=17)$ to a Wait-list condition (WLC, $n=18)$. Results showed a greater decrease in HR reactivity for CBT than for WLC. The change in HR reactivity was associated with clinical improvement.
\end{abstract}

KEY WORDS: heart rate; MVA; PTSD; treatment.

Psychophysiological research on posttraumatic stress disorder (PTSD) has shown both baseline (tonic) alterations and a heightened reactivity (phasic) to trauma-related stimuli. Heightened psychophysiological responsiveness to cues reminiscent to the trauma has been consistently shown for a variety of PTSD populations like combat veterans (Blanchard, Kolb, Taylor, \& Wittrock, 1989; Orr, Lasko, Shalev, \& Pitman, 1995; Orr et al., 2003; Orr, Meyerhoff, Edwards, \& Pitman, 1998), victims of childhood sexual abuse (McDonaghCoyle et al., 2001; Orr, Lasko et al., 1998), and in mixed trauma samples (Shalev, Orr, \& Pitman, 1993). In motor vehicle accident (MVA) survivors, Blanchard and colleagues (Blanchard, Hickling, Buckley et al., 1996; Blanchard, Hickling, Taylor, \& Loos, 1994;

\footnotetext{
${ }^{1}$ Department of Psychology, Dresden University of Technology, Germany.

${ }^{2}$ Roseneck Hospital, Prien am Chiemsee, Germany.

${ }^{3}$ Department of Abnormal Psychology, University of Zurich, Zurich, Switzerland.

${ }^{4}$ University of Southampton, School of Psychology, Southampton, UK.

${ }^{5}$ Address all correspondence to Sirko Rabe, Department of Psychology, Dresden University of Technology, Zellescher Weg 17, D-01069, Dresden, Germany; e-mail: rabe@biopsych.tu-dresden.de.
} 
Veazey, Blanchard, Hickling, \& Buckley, 2004) reported elevated psychophysiological responses to audiotaped idiosyncratic accident descriptions in PTSD patients compared to non-PTSD subjects with and without MVA. Especially heart rate (HR) reactivity has been shown to reliably discriminate diagnostic groups of MVA survivors (Blanchard, Hickling, Buckley et al., 1996; Blanchard et al., 1994; Veazey et al., 2004) or combat veterans (Blanchard et al., 1989; Casada, Amdur, Larsen, \& Liberzon, 1998; Keane et al., 1998). Results of a recent multisite study using a large sample of Vietnam veterans showed heightened baseline levels and increased physiological reactivity in response to both standardized audiovisual cues and idiosyncratic script driven imagery in PTSD participants (Keane et al., 1998).

Reports of baseline elevations of psychophysiological activity have been less consistent. In a recent meta-analysis, Buckley and Kaloupek (2001) demonstrated an association between PTSD and tonic cardiovascular activity (especially greater resting heart rate). Furthermore, they found that baseline heart rate was highest among the most chronic PTSD samples, which gave support for the assumption that repeated stress (cardiovascular responses) occurring over long periods of time causes cardiovascular dysfunction.

In study 1, we recorded heart rate activity during baseline and during the presentation of emotional pictures (neutral, positive, negative, trauma-related) in MVA victims with PTSD, subsyndromal PTSD, and without PTSD as well as in non-traumatized healthy controls. Based on previous reports on MVA victims we hypothesized that PTSD patients would show elevated HR reactivity specific to the trauma-related picture. Additionally, we obtained self report measures of affect. This was done for two reasons: first to evaluate the effects of baseline and emotional stimuli independently of HR, and second to assess the association of this subjective measures with HR to increase interpretability of our results. In Study 2 we examined whether altered HR patterns normalize with successful treatment. For this purpose we compared HR activity before and after cognitive behavioral therapy (CBT) in comparison to a Wait-list condition in a subsample of PTSD and subsyndromal PTSD patients of study 1.

\section{STUDY 1}

\section{Methods}

\section{Participants}

Seventy-three MVA survivors were recruited for a treatment study through self-referral local media coverage and advertising. It was required for inclusion that the accident was at least 6 months ago. Exclusion criteria were a history of neurological problems like epilepsy, brain surgery, and brain damage during the accident. Furthermore, we excluded subjects with current alcohol and/or substance abuse or dependence, current or past schizophrenic, bipolar, or psychotic disorder. All participants were required to be off all psychotropic medication for at least 1 month before testing.

Additionally, 28 healthy controls (HC) were recruited through local media coverage and advertising. They were required to have no history of any severe accident or other trauma and to have an absence of current or past DSM-IV axis I psychopathology. 


\section{Diagnostic Procedure}

After a short telephone screening asking for PTSD symptoms, exclusion criteria and details of the accident, participants were scheduled for the diagnostic and the psychophysiological assessments. All participants received a comprehensive description of the study and provided written informed consent at the initial diagnostic assessment. Both MVA subjects and $\mathrm{HC}$ were paid 15 Euro for undergoing the assessments. Participants were assessed by advanced doctoral and diploma students in clinical psychology who had previously received extensive training in the diagnostic procedures.

First, an accident-interview was conducted asking for details and information about severity of the accident and injuries. Second, a German version (Schnyder \& Moergeli, 2002) of the Clinician Administered PTSD Scale (CAPS) (Blake et al., 1995) was used to assess PTSD status and severity. The CAPS allows both, a categorical diagnosis of either PTSD, subsyndromal PTSD (sub-PTSD) or non-PTSD, and a sum CAPS score indicating PTSD severity. Reliability of PTSD diagnosis was established by rescoring (which was conducted by clinical psychologists blinded to the diagnosis) the CAPS of a subsample $(n=18)$ of interviews. This yielded a good diagnostic agreement (kappa of .824, $p<.001$ ). At the end of the session, the Structured Clinical Interview for the DSM-IV (SCID) (First, Spitzer, Gibbon, \& Williams, 1996; German version: Wittchen, Wunderlich, Gruschwitz, \& Zaudig, 1997) was conducted (except the PTSD module), evaluating the presence of concurrent and lifetime DSM-IV Axis I disorders.

In addition, a battery of self-report measures was sent to the participants that had to be filled in until the diagnostic session. Depression was assessed using a German version (Hautzinger, Bailer, Worall, \& Keller, 1994) of the Beck Depression Inventory (BDI) (Beck $\&$ Steer, 1987). State and trait anxiety were evaluated using the German version of the State-Trait Anxiety Inventory (STAI) (Laux, Glanzmann, Schaffner, \& Spielberger, 1981). Trait and state positive (PA) and negative (NA) affect were assessed by an extended (24items) German version (Krohne, Egloff, Kohlmann, \& Tausch, 1996) of the Positive and Negative Affect Schedule (PANAS) (Watson, Clark, \& Tellegen, 1988).

\section{Participant Characteristics}

The MVA victims were classified based on the CAPS into three groups: full PTSD, subsyndromal PTSD, and non-PTSD. Patients with full PTSD met symptomatic criteria for PTSD according to DSM-IV (symptom clusters B through D). Diagnosis of subsyndromal PTSD was given when participants met criterion B (Intrusion) and either Criterion C (Avoidance/Numbing) or Criterion D (Hyperarousal) following the most prominent definition of subsyndromal PTSD proposed by Blanchard and colleagues (e.g. Blanchard, Hickling, Taylor et al., 1996). Subsyndromal or partial PTSD has been shown to constitute a significant proportion of MVA victims that is clinical meaningful and associated with significant distress (Marshall et al., 2001; Schuetzwohl \& Maercker, 1999). The non-PTSD group was required to meet either none or one criterion but not criterion $\mathrm{F}$ (experience distress because of their PTSD symptoms).

This classification yielded three groups: $\operatorname{PTSD}(n=26)$, subsyndromal PTSD $(n=24)$, and non-PTSD $(n=23)$. The data sets of 2 subjects with subsyndromal PTSD, 3 with non-PTSD, and $1 \mathrm{HC}$ were excluded because of an insufficient amount of artifact free heart 
rate data. The resulting final sample comprised patients with PTSD $(n=26)$, subsyndromal PTSD $(n=22)$, non-PTSD controls with MVA $(n=20)$, and healthy controls $(n=27){ }^{6}$

\section{Heart Rate Assessment Procedure}

Experimental Conditions. The assessment of electrocardiograms (ECG) was conducted usually on the same day as the interview. After arriving at the laboratory, participants were asked to fill in the state version of the STAI. The assessment was conducted in an electrically shielded room with dim light and the participants sitting in a comfortable arm chair. After electrode application, a baseline data were collected. Participants were informed that there would be eight 1 min baselines, four with eyes open four with eyes closed and were asked to try to minimize eye blinks and movements. Two randomly assigned, counterbalanced orders were used for eyes open $(\mathrm{O})$ and eyes closed $(\mathrm{C})$ trials of the baselines (C-O-O-C-O-C-C-O, O-C-C-O-C-O-O-C). Participants heard a tone indicating the beginning and a double tone indicating the end of each 1-min recording. Immediately after the final baseline period the participant's mood was assessed with the PANAS-state questionnaire. The baseline recording procedure is recommended for reliable electroencephalographic (EEG) baseline measurement (Tomarken, Davidson, Wheeler, \& Kinney, 1992), which was also assessed in this study but is reported elsewhere (Rabe, Beauducel, Zöllner, Maercker, \& Karl, in press).

After the baseline assessment, 4 phases of mood induction via picture presentation were conducted. The positive (two bunnies), negative (a barking dog), and neutral (spoon) pictures were taken from the International Affective Picture System (IAPS) (Lang, Öhmann, \& Vaitl, 1988). The MVA-picture was an actual photograph from a crashed car lying on the roof. The sequence of the picture presentation phases was counterbalanced between participants. The picture presentation phases lasted $1 \mathrm{~min}$ and were separated by intervals of 2 min. Directly after picture presentations, participants rated their mood using the PANASstate questionnaire. Following this procedure, an event-related-potential experiment (not reported here) was administered.

Measurement of Heart Rate. ECG's were recorded with surface $\mathrm{Ag} / \mathrm{AgCl}$ electrodes attached to the right clavicle and the seventh rib on the left. The ECG signal was recorded by a Nihon Kohden amplifier (NeuroFileII system), filtered with a time constant (10 s), a high frequency cut-off $(300 \mathrm{~Hz})$ and was digitized online at $1024 \mathrm{~Hz}$ and stored at $256 \mathrm{~Hz}$. Additionally, we recorded electroencephalographic (EEG) activity. ECG data were edited with a customized computer program developed in our laboratory. Heart rate was extracted by triggering $\mathrm{R}$ waves and measuring the time between $\mathrm{R}$ waves. ECG data were visually inspected for artifacts. Artifactual HR data points (not correctly triggered R waves) were replaced by manually included trigger points. Data sets with high proportions of artifacts were not evaluated.

\footnotetext{
${ }^{6}$ Based on the SCID interview, 5 PTSD patients fulfilled criteria for a lifetime major depression (MDD) but not current, 10 for current MDD. Additional diagnoses in the PTSD group were current dysthymia $(n=2)$, panic disorder without agoraphobia $(n=2)$; panic disorder with agoraphobia $(n=1)$, agoraphobia without panic disorder $(n=2)$, social phobia $(n=2)$, specific phobia $(n=6)$, lifetime generalized anxiety disorder $(n=1)$, and obsessive compulsive disorder $(n=1)$. Additional diagnoses in the subsyndromal PTSD group were MDD $(n=4)$, social phobia $(n=3)$, and specific phobia $(n=1)$. Lifetime but not current diagnoses in the non-PTSD group were $\operatorname{MDD}(n=5)$, panic disorder without agoraphobia $(n=1)$, social phobia $(n=1)$. One of the nonPTSD participants met the criteria for mild current specific phobia.
} 
Response Definitions. The mean values of HR for the 8 one-minute baselines were averaged to yield a single value for baseline HR. For calculation of HR reactivity scores $(\triangle \mathrm{HR})$ during picture presentation we first computed mean values of HR for each of the four 1-min pictures. Then, we subtracted the value for the neutral picture from the value for the positive, negative, and trauma-related pictures. This resulted in HR-reactivity values $(\triangle \mathrm{HR})$ for the positive, negative, and trauma-related pictures.

\section{Results}

\section{Demographics and Psychometrics}

Demographic and psychometric data were examined using Chi-Square tests and oneway ANOVAs with Group (PTSD, sub-PTSD, non-PTSD, HC) as between-subjects variable followed by Tukey multiple comparison tests. As summarized in Table I, there were no significant differences between the diagnostic groups in gender, age, and time since the accident. PTSD patients had significantly higher scores in PTSD severity (CAPS Score), depression (BDI), and trait anxiety (STAI) than the other 3 groups. Subsyndromal PTSD patients had higher scores on the same measures than the 2 control groups, who did not differ in BDI and STAI-Trait.

\section{Baseline}

Baseline Negative Affect. One-way ANOVA for baseline PANAS-NA revealed a significant Group effect $\left[F(3,90)=6,55, p<.001, \eta^{2}=.179\right]$. Tukey multiple comparison tests

Table I. Diagnostic and Demographic Characteristics by Diagnostic Group

\begin{tabular}{|c|c|c|c|c|c|c|}
\hline Characteristics & PTSD & Sub- PTSD & $\begin{array}{l}\text { Non-PTSD } \\
\text { with MVA }\end{array}$ & $\begin{array}{l}\text { Healthy } \\
\text { controls }\end{array}$ & Test & $p$ \\
\hline$N$ & 26 & 22 & 20 & 27 & & \\
\hline \multicolumn{7}{|c|}{ Gender (male/female) } \\
\hline$N$ & $6 / 20$ & $7 / 15$ & $9 / 11$ & $7 / 20$ & $\chi^{2}(3)=2.94$ & .40 \\
\hline$\%$ Female & 77 & 68 & 57 & 74 & & \\
\hline \multicolumn{7}{|l|}{ Age (years) } \\
\hline$M$ & 43.12 & 36.09 & 44.10 & 38.56 & $F(3,91)=2.27$ & .09 \\
\hline$S D$ & 11.10 & 9.79 & 16.33 & 10.27 & & \\
\hline Months since MVA & & & & - & & \\
\hline$M$ & 72.65 & 38.64 & 69.60 & & $F(2,65)=1.21$ & .30 \\
\hline$S D$ & 96.99 & 36.15 & 93.56 & & & \\
\hline CAPS score & & & & - & & \\
\hline$M$ & 57.50 & 32.95 & 6.05 & & $F(2,65)=169.29$ & $<.001$ \\
\hline$S D$ & 10.93 & 10.61 & 4.64 & & & \\
\hline \multicolumn{7}{|l|}{ Depression (BDI) } \\
\hline M & 22.88 & $12.10^{a}$ & $5.68^{b}$ & 3.81 & $F(3,89)=38.10$ & $<.001$ \\
\hline$S D$ & 9.11 & 7.75 & 5.51 & 4.59 & & \\
\hline \multicolumn{7}{|l|}{ Trait anxiety (STAI) } \\
\hline$M$ & 55.85 & $44.81^{a}$ & $35.00^{b}$ & 35.19 & $F(3,89)=27.94$ & $<.001$ \\
\hline$S D$ & 10.68 & 8.89 & 9.53 & 7.85 & & \\
\hline
\end{tabular}

Note. BDI = Beck Depression Inventory Depression, STAI-Trait = State-Trait Anxiety Inventory-Trait Scale, CAPS $=$ Clinician-Administered PTSD Scale.

$a_{n=21 \text {. }}$

${ }^{b} n=19$. 
indicated that PTSD patients reported significantly more negative affect than non-PTSD subjects $(p<.01)$ and healthy controls $(p<.001)$ but did not differ from the group with subsyndromal PTSD $(p=.25)$. There was no significant statistical difference between the three groups: subsyndromal PTSD, non-PTSD, and healthy controls (all $p>.15$ ).

Heart Rate. The one-way ANOVA for baseline HR showed a tendency for the Group effect $\left[F(3,90)=2.54, p=.06, \eta^{2}=.078\right]$. Tukey multiple comparison tests showed that the PTSD group had a significant trend of higher baseline heart rate compared to non-PTSD controls $(p=.08)$. For all other multiple comparisons $p>.14$ was revealed.

Correlational analysis revealed that PTSD severity (CAPS Score) was positively related to baseline heart rate $[r(n=67)=.27, p<.05]$. It has been hypothesized that baseline heart rate alterations in PTSD may be explained by chronicity of the disorder or state affect and test anxiety (Buckley \& Kaloupek, 2001). Accordingly, we computed two-tailed Pearson product-moment correlations between baseline heart rate and the time since accident (in months), state anxiety (STAI) measured before the experiment, and PANAS-NA for the baseline. There was a significant association of baseline heart rate with PANAS-NA $[r(n=94)=.26, p<.05]$ but not with time since accident and state anxiety (both $p>.40$ ). Thus, the more negative the baseline condition was rated the higher was heart rate during this condition. In an analysis of covariance (ANCOVA) we repeated the above analysis on baseline HR using baseline PANAS-NA as covariate. This resulted in a decrease of the group effect on baseline heart rate $[F(3,89)=1.29, p=.28]$ indicating that negative affect during baseline might mediate the association of PTSD diagnosis and baseline heart rate.

\section{Mood Conditions}

Negative Affective Responses. A two-way repeated measures Group $\times$ Condition (neutral, positive, negative, trauma-related) ANOVA was computed for PANAS-NA scores. This analysis revealed significant main effects for Group $[F(3,91)=5.26, p<.01$, $\left.\eta^{2}=.148\right]$, Condition $\left[F(3,91)=124.86, p<.001, \eta^{2}=.578\right]$, and a significant two-way interaction Group $\times$ Condition $\left[F(9,273)=6.08, p<.001, \eta^{2}=.167\right]$. Follow-up one-way ANOVAs for each condition separately revealed that there were no significant group differences for the neutral, positive, and negative pictures (all $p>.27$ ). However, for the trauma-related condition there was a significant Group effect $[F(3,91)=11,58, p<.001$, $\left.\eta^{2}=.276\right]$. Tukey multiple comparison tests revealed that the PTSD group differed from the two control groups (all $p<.01$ ) in NA but not from subsyndromal PTSD subjects $(p=.14)$. Furthermore, subsyndromal PTSD subjects displayed more negative affect than the non-PTSD controls $(p<.01)$ but did not differ from healthy controls $(p=.49)$. The two control groups did not differ significantly $(p=.13)$ in NA for the trauma-related condition.

Heart Rate Reactivity. We first computed a two-way repeated measures of variance (ANOVA) on heart rate reactivity scores with Condition (positive, negative, trauma-related) as the within-subject variable and Group (PTSD, subsyndromal PTSD, non-PTSD, HC) as between-subject-factor. There was a main effect for Condition $[F(2,90)=5.20, p<.01$, $\left.\eta^{2}=.104\right]$ but no main effect for Group $[F(3,91)=1.97, p=.12]$. As expected there was a significant Group $\times$ Condition interaction $\left[F(6,182)=2.25, p<.05, \eta^{2}=.069\right]$. These data are displayed in Fig. 1. As can be seen there was an increase in heart rate during the trauma-related condition for PTSD and subsyndromal PTSD participants in comparison to the two control groups. Follow-up one-way ANOVAs (see Table II) for each condition 


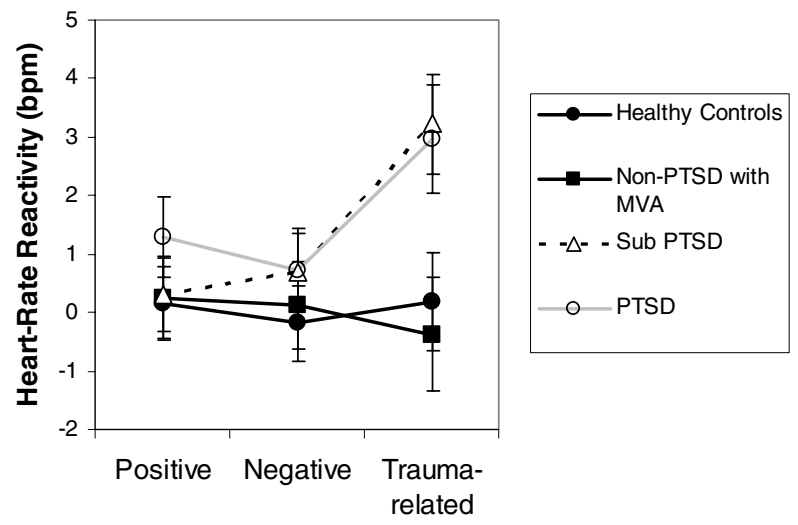

Fig. 1. Mean heart rate reactivity scores (HR emotional picture minus HR neutral picture) in participants with PTSD, subsyndromal PTSD (sub-PTSD) as well as non-PTSD controls with MVA and healthy controls. Error bars represent standard errors of the mean.

separately showed that there were no significant group differences in heart rate reactivity for the positive and negative picture (both $p>.60$ ). However, for the trauma-related picture there was a significant Group effect $\left[F(3,91)=4.21, p<.01, \eta^{2}=.122\right]$. Tukey multiple comparison tests indicated that HR reactivity for the trauma-related picture was significantly higher in PTSD patients as compared to non-PTSD group $(p<.04)$ and a tendency when compared to HC ( $p=.06)$ but did not differ from subsyndromal PTSD patients $(p=.99)$. Subsyndromal PTSD subjects showed a trend for significantly higher heart-rate reactivity in comparison to non-PTSD participants $(p=.07)$, but not healthy controls $(p=.13)$. The two control groups did not differ significantly $(p=.97)$.

It has been shown that heart rate reactivity scores might be used to discriminate diagnostic groups. Therefore we used the same cutoff score of $+2 \mathrm{bpm}$ or greater as previously reported in MVA victims (Blanchard, Hickling, Buckley et al., 1996; Blanchard et al., 1994; Veazey et al., 2004). Using this cutoff score we found a sensitivity of 50\% (13 out of 26 correctly identified PTSD patients). The specificity (correctly identified as not having PTSD) was .90 for the non PTSD group with MVA, .78 for non-MVA HC, and .81 for the combined sample of control subjects. This resulted in classification rates of correctly

Table II. Initial Assessment of Heart Rate Values for Baseline and Reactivity by Diagnostic Group

\begin{tabular}{|c|c|c|c|c|c|c|}
\hline $\begin{array}{l}\text { Baseline HR/HR } \\
\text { Reactivity }(\Delta \mathrm{HR})\end{array}$ & PTSD & Sub-PTSD & $\begin{array}{l}\text { Non-PTSD } \\
\text { with MVA }\end{array}$ & $\begin{array}{l}\text { Healthy } \\
\text { Controls }\end{array}$ & $\begin{array}{l}\text { One-way } \\
\text { ANOVA }\end{array}$ & $p$ \\
\hline Baseline HR & $76.24(9.00)^{a}$ & $70.89(8.18)$ & $70.65(6.48)$ & $70.42(10.18)$ & $F(3,90)=2.54$ & .06 \\
\hline$\Delta$ HR Positive Picture & $.30(4.00)$ & $1.30(3.07)$ & $0.25(3.00)$ & $0.16(2.65)$ & $F(3,91)=.62$ & .60 \\
\hline$\Delta \mathrm{HR}$ Negative Picture & $0.70(4.85)$ & $0.72(2.63)$ & $0.12(1.93)$ & $-0.18(2.81)$ & $F(3,91)=0.44$ & .72 \\
\hline $\begin{array}{l}\Delta \text { HR Trauma-related } \\
\text { Picture }\end{array}$ & $3.23(6.47)$ & $2.96(4.65)$ & $-0.38(1.84)$ & $0.18(2.61)$ & $F(3,91)=4.21$ & $<.01$ \\
\hline
\end{tabular}

Note. $\triangle \mathrm{HR}$, Heart Rate Reactivity.

$a_{n=25}$. 
classified subjects of 67\% (PTSD vs. non-PTSD with MVA), 64\% (PTSD vs. non-MVA HC) and $71 \%$ (PTSD vs. non-PTSD and HC).

Finally, we computed two-tailed Pearson product-moment correlations between heart rate reactivity measures and PTSD severity and ratings of negative affect during picture presentation for the combined samples. Only HR reactivity during presentation of the trauma-related picture was positively related to PTSD severity $[r(n=67)=.25, p<.05]$ and NA during this condition $[r(n=95)=.34, p<.001]$.

\section{Discussion}

The present study investigated baseline heart rate and heart rate reactivity to traumarelated, non-trauma-related negative, and positive pictures in MVA survivors with PTSD, subsyndromal PTSD, and without PTSD as well as non-traumatized healthy controls. For baseline HR we found elevated values in PTSD subjects with an average of approximately $6 \mathrm{bpm}$ faster relative to the other 3 groups. These results are similar to that reported in a recent meta-analysis (Buckley \& Kaloupek, 2001), although studies in MVA related PTSD did not find elevated basal HR (Blanchard, Hickling, Buckley et al., 1996; Blanchard et al., 1994; Veazey et al., 2004). Furthermore, PTSD patients rated the baseline condition as more aversive (PANAS-NA). Correlational analysis revealed that baseline HR was significantly associated with PTSD severity and state NA experienced during the baseline but not with the time since accident or anticipatory anxiety (STAI-state). Furthermore, when controlling for baseline state negative affect, group differences in baseline HR were no more significant.

HR reactivity was higher in PTSD and subsyndromal PTSD patients in comparison to non-PTSD and healthy controls but only in response to the trauma-related picture but not to positive or non-trauma-related negative pictures. Furthermore, there was a significant correlation between HR reactivity to the trauma-related picture and experienced negative affect during this condition.

\section{STUDY 2}

There are only few studies that have investigated changes in physiological reactivity in PTSD patients due to therapeutic interventions. Boudewyns and Hyer (1990) compared direct therapeutic exposure (DTE) with conventional counseling in male Vietnam veterans with PTSD using skin conductance level (SCL) and HR to imagery exposure to scenes of stressful combat memories. They did not find significant differences between treatment conditions in reduction of physiological responses. However, for HR there was a trend of diminished HR-reactivity in DTE but not controls. Shalev, Orr, and Pitman (1992) investigated SCL, HR, and frontalis electromyographic (EMG) responses during scriptdriven imagery as an outcome measure in 3 PTSD patients before and after treatment with systematic desensitization. Physiological responses to imagery of traumata normalized with successful treatment. However, the lack of a control group impairs interpretation of the differential impact of treatment on physiological responses. Karl, Malta, Alexander, and Blanchard (2004) investigated startle responses in a small sample of MVA survivors with PTSD and subsyndromal PTSD as part of a controlled treatment study comparing (CBT), Supportive Therapy (ST), and a Wait-list condition. At posttreatment, a significant 
reduction in startle reactivity to different stimuli was observed in participants who received active treatment (either CBT or ST), compared to Wait-list controls.

Recently, Blanchard et al. (2002) investigated MVA survivors with PTSD or subsyndromal PTSD, who completed a randomized, controlled trial with three conditions: CBT, supportive Therapy (SP), and Wait-list. They measured HR reactivity to audiotaped idiosyncratic accident descriptions, both before and after each of the treatments. Results showed a greater reduction in HR reactivity for CBT compared to SP or Wait-list. Furthermore, they found a significant correlation between PTSD severity changes and changes in HR reactivity.

The aim of study 2 was to examine whether heightened reactivity to the trauma-related picture and increased baseline HR observed in study 1 normalizes due to successful treatment. Therefore, we compared HR data in MVA survivors with PTSD and subsyndromal PTSD assessed before and after CBT in comparison to Wait-list controls (WLC), as part of a recently completed randomized, controlled treatment trial (Maercker, Zollner, Menning, Rabe, \& Karl, 2006). ${ }^{7}$ We expected a greater reduction in HR reactivity to the traumarelated picture for the CBT group than for the WLC. Furthermore, we hypothesized that a reduction in PTSD severity over time is related to a reduction in HR reactivity.

\section{Methods}

The procedure for measurement of ECG activity during baseline and mood induction was described earlier under Study 1 and identical between pre- and post-assessments. Complete interview data and self-report measures were nearly identical to pre-treatment assessment with exception of the CAPS. At post-assessment we used a German version of the CAPS specifically designed for reassessments (CAPS-2) (Blake et al., 1990) asking for severity of PTSD symptoms during the last week.

\section{Participants}

The participants in study 2 are a subset of the participants of study 1 . As described elsewhere (Maercker et al., 2006) 42 participants with PTSD or subsyndromal PTSD completed the treatment trial and pre- and post-assessments. For the ECG analyses 5 were excluded from the current study because they did not meet the inclusion criterion to be off psychotropic medication off all psychotropic medication for at least 1 month before the pre-treatment assessment. Two participants were excluded because of ECG recording errors or an insufficient amount of artifact free ECG data. This resulted in a final sample of 35 patients with PTSD $(n=17)$ or subsyndromal PTSD $(n=18)$. Demographic variables for the participants in each of the treatment conditions are presented in Table III. The CBT and WLC groups did not differ on any of the variables except gender. There were fewer men than women in the CBT group compared to the WLC. ${ }^{8}$

\footnotetext{
${ }^{7}$ The article by Maercker et al. (2006) gives a full description of the randomized, controlled treatment trial and does contain only subjective and interview data but not psychophysiological data.

${ }^{8}$ Comorbid diagnoses in the CBT group were lifetime but not current MDD $(n=3)$, current $\operatorname{MDD}(n=6)$, panic disorder without agoraphobia $(n=1)$, agoraphobia without panic disorder $(n=1)$, social phobia $(n=3)$, specific phobia $(n=1)$, obsessive compulsive disorder $(n=1)$, and lifetime generalized anxiety disorder $(n=1)$.
} 
Table III. Diagnostic and Demographic Characteristics by Treatment Condition

\begin{tabular}{|c|c|c|c|c|c|}
\hline Characteristics & CBT & WLC & Total & Test & $p$ \\
\hline$N$ & 17 & 18 & 35 & & \\
\hline \multicolumn{6}{|l|}{ Gender (male/female) } \\
\hline$N$ & $2 / 15$ & $8 / 10$ & $10 / 25$ & \multirow[t]{2}{*}{$\chi^{2}(1)=4.58$} & \multirow[t]{2}{*}{$<.05$} \\
\hline$\%$ Female & 87 & 56 & 71 & & \\
\hline \multicolumn{6}{|l|}{ Age (years) } \\
\hline$M$ & 38.65 & 41.89 & 40.31 & \multirow[t]{2}{*}{$t(33)=-0.85$} & \multirow[t]{2}{*}{.400} \\
\hline$S D$ & 11.47 & 11.03 & 11.20 & & \\
\hline \multicolumn{6}{|l|}{ Months since MVA } \\
\hline$M$ & 74.18 & 41.39 & 57.31 & \multirow[t]{2}{*}{$t(33)=1.17$} & \multirow[t]{2}{*}{.25} \\
\hline$S D$ & 114.61 & 32.77 & 83.64 & & \\
\hline \multicolumn{6}{|l|}{ Pretreatment diagnoses } \\
\hline PTSD & 10 & 7 & 17 & \multirow{2}{*}{$\chi^{2}(1)=1.39$} & \multirow[t]{2}{*}{.24} \\
\hline sub-PTSD & 7 & 11 & 18 & & \\
\hline \multicolumn{6}{|c|}{ Pretreatment CAPS Score } \\
\hline$M$ & 46.94 & 41.39 & 44.09 & \multirow[t]{2}{*}{$t(33)=0.98$} & \multirow[t]{2}{*}{.34} \\
\hline$S D$ & 16.13 & 17.45 & 16.81 & & \\
\hline \multicolumn{6}{|l|}{ Depression (BDI) } \\
\hline$M$ & 16.94 & 15.67 & 16.29 & \multirow[t]{2}{*}{$t(33)=0.37$} & \multirow[t]{2}{*}{.70} \\
\hline$S D$ & 10.59 & 8.90 & 9.63 & & \\
\hline \multicolumn{6}{|l|}{ Trait Anxiety (STAI) } \\
\hline$M$ & 49.29 & 49.17 & 49.23 & \multirow[t]{2}{*}{$t(33)=0.03$} & \multirow[t]{2}{*}{.97} \\
\hline$S D$ & 10.47 & 12.51 & 11.39 & & \\
\hline
\end{tabular}

Note . $\mathrm{CBT}=$ Cognitive Behavioral Therapy, BDI $=$ Beck Depression Inventory Depression, STAI-Trait, State-Trait Anxiety Inventory-Trait Scale, CAPS = Clinician-Administered PTSD Scale.

\section{Treatment Trial}

A full description of the randomized, controlled treatment trial has been published recently (Maercker et al., 2006). After the initial assessment, patients were matched into dyads based on CAPS score, age, and comorbidity and then randomly assigned to one of two conditions: (1) a combination of cognitive behavioral treatment (CBT) procedures or (2) WLC. The CBT represents a German adaptation and extension of the CBT manual of Hickling and Blanchard (1997) and has been published recently (Zöllner, Karl, Maercker, Hickling, \& Blanchard, 2005). It includes standard CBT techniques for the treatment of PTSD such as imaginal exposure, in-vivo exposure, relaxation training, and cognitive restructuring. Additionally, it contains sections for the treatment of feelings of guilt or anger and a section on facilitation of posttraumatic growth. The CBT included a range of 8-12 weekly sessions with an expected mode of 10 . Six therapists were trained for the CBT manual and were supervised by one of the co-authors (A.M.) and three other licensed and experienced therapists.

The WLC were told that their treatment would be delayed for 2-3 months and that they would need to be reassessed after that interval before beginning therapy. After the post-treatment assessment, they were all given the CBT if they were still interested in treatment. 


\section{Results}

\section{Treatment Outcome}

For assessment of treatment outcome we calculated a two-way repeated measures ANOVA on the CAPS score with Time (Pre, Post) as within-subject factor and TreatmentGroup (CBT, WLC) as between-subject-factor. This analysis revealed a significant main effect of Time $\left[F(1,33)=59.47, p<.001, \eta^{2}=.643\right]$ and a significant interaction of Group $\times$ Time $\left[F(1,33)=25.32, p<.001, \eta^{2}=.434\right]$ but no main effect of TreatmentGroup. A between group comparison at post-test by ANCOVA (controlled for pre-Caps values) revealed that the CBT group showed significantly greater change than the WLC group $\left[F(1,32)=23.49, p<.001, \eta^{2}=.432\right]$.

\section{Baseline}

Baseline Affect. We repeated the above ANOVA for baseline PANAS-NA scores. There were no significant main effects or interactions with Time or Treatment-Group (all $p>.18)$.

Heart Rate. The ANOVA revealed a significant main effect for Time $[F(1,33)=9.97$, $\left.p<.01, \eta^{2}=.232\right]$ but not Treatment-Group $(p=.19)$ and no significant TreatmentGroup $\times$ Time interaction $(p=.86)$. In both groups baseline heart rate decreased. Correlational analysis also showed that a reduction in basal heart rate was not significantly related to change in CAPS score $[r(35)=.14, p=.44]$ or PANAS-NA $[r(35)=.04, p=$ $.82)$.

\section{Mood Conditions}

Affective Responses. To evaluate treatment-related changes in affective responses during the different conditions we calculated a three-way ANOVA on PANAS-NA scores with Time (Pre, Post) and Condition (neutral, positive, negative, trauma-related) as within-subject variables and Treatment-Group (CBT, WLC) as between-subject-factor. There were significant main effects for Time $\left[F(1,33)=13.54, p<.001, \eta^{2}=.291\right]$, Condition $\left[F(1,33)=61.93, p<.001, \eta^{2}=.652\right]$, and a significant Time $\times$ Condition interaction $\left[F(3,99)=4.77, p<.01, \eta^{2}=.126\right]$ indicating differential decrease in NA over time depending on the condition. However, there was no main effect or interaction with Treatment-Group (all $p>.16$ ). Follow up ANOVAs (Treatment-Group $\times$ Time) for each condition separately revealed significant $(p<.05)$ Time effects for the negative and trauma-related pictures indicating a decrease of NA in both groups, but no significant interactions Treatment-Group $\times$ Time $(p<.20)$

Heart Rate Reactivity. Three-way ANOVA (Treatment-Group $\times$ Time $\times$ Condition) on heart-rate reactivity scores (mood condition minus neutral condition) revealed significant main effects for Time $\left[F(1,33)=6.60, p<.05, \eta^{2}=.167\right]$ and Condition $[F(2,32)=5.79$, $\left.p<.01, \eta^{2}=.149\right]$ and a significant Time $\times$ Condition interaction $[F(2,66)=4.78, p<.05$, $\left.\eta^{2}=.127\right]$. Furthermore, there was a tendency for the three-way interaction TreatmentGroup $\times$ Time $\times$ Condition $\left[F(2,66)=2.63, p=.09, \eta^{2}=.074\right]$ indicating condition- 
Table IV. Pre- and Posttreatment Values for Diagnoses, CAPS Score, Baseline-HR, and HR Reactivity to the Trauma-related Picture

\begin{tabular}{|c|c|c|c|c|c|c|}
\hline \multirow[b]{2}{*}{ Characteristics } & \multicolumn{2}{|c|}{ CBT } & \multicolumn{2}{|c|}{ WLC } & \multirow{2}{*}{$\begin{array}{l}\text { Interaction } \\
\text { Group } \times \text { Time }\end{array}$} & \multirow[b]{2}{*}{$p$} \\
\hline & PRE & POST & PRE & POST & & \\
\hline CAPS Score & 46.94 (16.13) & $15.24(12.99)$ & $41.39(17.45)$ & $34.72(22.14)$ & $F(1,33)=25.32$ & $<.001$ \\
\hline Baseline-HR & $71.21(11.29)$ & $67.55(10.51)$ & $75.05(9.25)$ & $71.81(6.99)$ & $F(1,33)=.04$ & .85 \\
\hline $\begin{array}{l}\Delta \text { HR positive } \\
\text { Picture }\end{array}$ & $1.71(2.62)$ & $0.13(1.48)$ & $1.26(3.08)$ & $1.14(2.12)$ & $F(1,33)=2.65$ & .21 \\
\hline $\begin{array}{l}\Delta \mathrm{HR} \text { negative } \\
\text { Picture }\end{array}$ & $2.11(4.61)$ & $-.16(2.21)$ & $1.10(3.23)$ & $0.99(3.36)$ & $F(1,33)=2.32$ & .14 \\
\hline $\begin{array}{l}\Delta \text { HR trauma- } \\
\text { related } \\
\text { Picture }\end{array}$ & $5.25(7.78)$ & $-0.11(2.40)$ & $2.62(4.15)$ & $1.98(3.06)$ & $F(1,33)=5.50$ & $<.05$ \\
\hline
\end{tabular}

Note. $\mathrm{CBT}=$ Cognitive Behavioral Therapy, $\triangle \mathrm{HRD} \Lambda$ Heart Rate Reactivity, CAPS $=$ Clinician-Administered PTSD Scale.

dependent pre/post treatment-group differences in heart-rate-reactivity. These data are displayed in Table IV.

Since our initial hypothesis asked for treatment related changes mainly during the trauma-related condition we calculated ANOVAs (Treatment-Group $\times$ Time) for each condition separately. For the positive and negative conditions there were no significant main effects or interactions (all $p>.10$ ). For the trauma-related condition there was a main effect for Time $\left[F(1,33)=8.88, p<.01, \eta^{2}=.212\right]$. Furthermore, the expected Time $\times$ Treatment-Group interaction reached significance $[F(1,33)=5.50, p<.05$, $\left.\eta^{2}=.143\right]$ indicating a greater decrease in the CBT group as compared to WLC (see Fig. 2). A between group comparison at post-test by ANCOVA (controlled for pre-values) revealed that the CBT group showed a greater change in HR reactivity than WLC $[F(1,32)=7.04$, $\left.p<.05, \eta^{2}=.18\right]$.

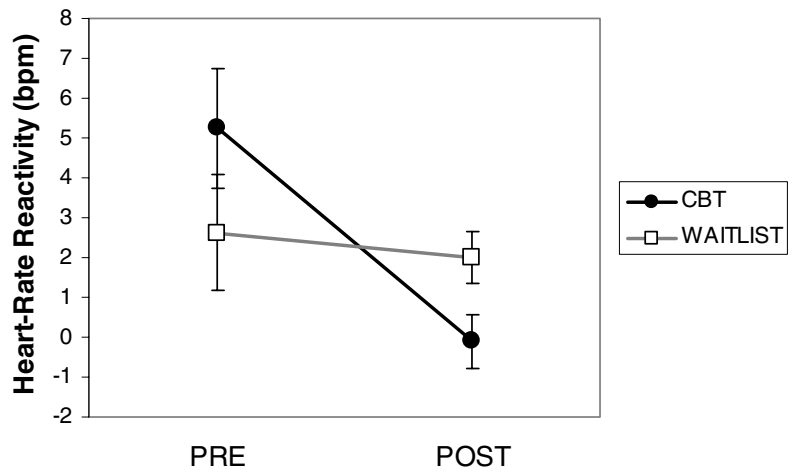

Fig. 2. Mean heart rate reactivity scores for the trauma-related picture (HR trauma-related picture minus HR neutral picture) for the two treatment groups at pre- and posttreatment. $\mathrm{CBT}=$ cognitive behavioral therapy. 
Another important question was how the change in HR reactivity is related to clinical improvement. Following previous research on this question (Blanchard et al., 2002) we therefore calculated correlation coefficients between changes in HR reactivity for the trauma-related condition and changes in CAPS score for combined groups of CBT and WLC. As predicted, there was a positive correlation $[r(35)=.30, p<.05$, one-tailed). A greater reduction in PTSD-symptoms was associated with a greater reduction in heart-ratereactivity to the trauma-related picture. For the other conditions no significant correlations were revealed. Furthermore, only for the trauma-related condition there was a trend for a significant correlation $[r(35)=.23, p=.09$, one-tailed) of change in HR reactivity with reduction in PANAS-NA.

\section{Discussion}

The aim of study 2 was to investigate whether heightened HR responses to the traumarelated picture observed in study 1 in participants with chronic PTSD or subsyndromal PTSD remit after successful treatment. For baseline HR there was a reduction in both CBT and WLC of about $4 \mathrm{bpm}$, which was not significantly related to clinical improvement or reduction in negative affect during baseline. This reduction may reflect a habituation to the laboratory situation. Consistent with the results of Blanchard et al. (2002) we found a significant greater decrease of HR reactivity to the trauma-related picture for the CBT group than for the WLC group. The CBT group showed essentially no heightened reactivity at post-assessment, which was comparable to the responses of non-PTSDs and healthy controls in study 1 . Furthermore, the decrease in HR reactivity was positively associated with reduction in PTSD severity as measured with the CAPS. Thus, clinical change as measure by the CAPS is in part mirrored by an "objective" physiological measure. These effects were trauma specific. However on a descriptive level, as can be seen in Table IV, the changes of HR reactivity scores for the positive and negative pictures paralleled that of the trauma-related picture.

\section{General Discussion}

The results from study 1 showed that baseline HR was increased in PTSD patients only, who also showed an increased negative affect during baseline. Sitting in a dark relatively small room may itself be reminiscent of the accident and therefore more aversive for PTSD patients. The results of study 2 showed that baseline HR decreased similarly in CBT and WLC and was not related to clinical improvement. The results let us speculate that heightened baseline HR in PTSD patients with MVA may reflect an acute emotional reaction (but not chronic hyperarousal) which simply habituates with repeated measurement. However, a definitive interpretation of our results is not possible. Increased negative affect and heightened heart rates of the PTSD patients might be present during different situations outside the laboratory and thus chronic. A longtime assessment of HR (e.g. $24 \mathrm{hr}$ ) in different natural situations might be useful to answer that question.

As was shown in study 1, MVA survivors with chronic PTSD and subsyndromal PTSD showed higher HR reactivity specific to a trauma-related picture. This is consistent with findings in MVA-related PTSD (Blanchard, Hickling, Buckley et al., 1996; Blanchard 
et al., 1994; Veazey et al., 2004) and other PTSD subpopulations like combat veterans (e.g., Keane et al., 1998) or sexually abused women (e.g., Orr, Lasko et al., 1998). HR reactivity was not different between groups for positive or non-trauma-related negative pictures which underlines that group differences in HR reactivity are not attributable to a generalized hyperarousal to a stimulus change, but are specific only to trauma-related cues (e.g., Casada et al., 1998).

The average magnitude of HR reactivity of about $3 \mathrm{bpm}$ was smaller than that reported for ideosyncratic audiotaped MVA descriptions ranging from 4-7 bpm (e.g., Blanchard, Hickling, Buckley et al., 1996; Veazey et al., 2004). Furthermore, classification rates based on HR reactivity were lower than those previously reported in MVA-victims, but comparable to rates observed in a large study of combat-related PTSD (Keane et al., 1998). Especially, the sensitivity for PTSD diagnosis was reduced with $50 \%$ of non-responding PTSD participants in contrast to a good specificity. One reason for this might be that we used standardized pictures instead of idiosyncratic descriptions of the MVAs.

HR reactivity to the trauma-related picture in the subsyndromal PTSD group was comparable to PTSD patients. This is in contrast to earlier studies with MVA victims (e.g., Blanchard, Hickling, Buckley et al., 1996; Veazey et al., 2004). One explanation for this finding might be that our sample of subsyndromal PTSD participants was more chronic with an average of 38 months post accident in comparison to earlier studies in MVA victims with an average of 1-12 months since accident. Initial acute PTSD may remit to subsyndromal PTSD (Blanchard, Hickling, Barton et al., 1996) and it might be reasonable to assume that persons who suffer chronically from PTSD symptoms differ from those with more recent onset of symptoms. Future studies should explore the differences between acute and chronic subsyndromal PTSD.

Study 2 attempted to replicate the findings of Blanchard et al. (2002) who showed a reduction in HR reactivity for PTSD and subsyndromal PTSD patients receiving a CBT treatment. Similarly, we found a greater decrease in HR reactivity to the trauma-related picture in the CBT group than for WLC controls. Psychological treatment models of PTSD propose that activation of the traumatic fear memories via imaginal exposure is crucial for successful treatment (Foa \& Rothbaum, 1998). However, future studies are needed to investigate which components of CBT (e.g. imaginal exposure, cognitive restructuring, relaxation procedures) may be essential for the reduction of HR responses.

Comparable to the study of Blanchard et al. the reduction in HR reactivity accounted only for little variance in CAPS score changes. There are several explanations for this finding. First, as mentioned by Blanchard et al., clinical status of PTSD is a multi-determined phenomenon and change in "objectively" measured physiological arousal represents only one facet of change. Second, repeated HR measurement using identical stimulus material at pre- and post-assessments may lead to a reduction of HR responses simply due to habituation. Future studies investigating treatment related physiological changes might use different stimuli at pre- and post-assessments. This may increase the reliability of measured HR responses and increase validity of HR measurement as a diagnostic tool reflecting clinical improvement, although in repeated psychophysiological assessments there will be always some habituation to the testing environment.

In summary, we demonstrated trauma-specific exaggerated HR reactivity in PTSD and subsyndromal PTSD in contrast to MVA controls and non-MVA controls, which normalized with successful treatment. 


\section{ACKNOWLEDGMENTS}

This study was supported by the Deutsche Forschungsgemeinschaft (KA 1476/3). We thank Ullrich Buhss for help with data analysis and the many students who helped with data collection. The authors would like to thank Edward B. Blanchard and Edward J. Hickling for many helpful suggestions at various stages of our treatment study. We would also like to thank our colleagues Anne Boos, Andrea Hähnel, and Michael Klose for serving as therapists, and Silvia Lemke, Andreas Poldrack, and Frank Schirmer for supervisory activities. We also have to thank Peter Dettmar, Clemens Kirschbaum, HansUlrich Wittchen, and Jürgen Hoyer for their support with access to laboratory and treatment facilities at the University of Technology Dresden.

\section{REFERENCES}

Beck, A. T., \& Steer, R. A. (1987). Beck depression inventory. San Antonio, TX: The Psychological Cooperation.

Blake, D. D., Weathers, F. W., Nagy, L. M., Kaloupek, D. G., Charney, D. S., \& Keane, T. M. (1995). Clinicianadministered PTSD scale for DSM-IV (CAPS-DX). Boston, MA.

Blake, D. D., Weathers, F. W., Nagy, L. M., Kaloupek, D. G., Klauminzer, G. W., Charney, D. S., et al. (1990). Clinician-administered PTSD scale for DSM-IV (CAPS), Form 2 one-week symptom status version. Boston, MA.

Blanchard, E. B., Hickling, E. J., Barton, K. A., Taylor, A. E., Loos, W. R., \& Jones-Alexander, J. (1996). One-year prospective follow-up of motor vehicle accident victims. Behaviour Research and Therapy, 34(10), 775-786.

Blanchard, E. B., Hickling, E. J., Buckley, T. C., Taylor, A. E., Vollmer, A., \& Loos, W. R. (1996). Psychophysiology of posttraumatic stress disorder related to motor vehicle accidents: Replication and extension. Journal of Consulting and Clinical Psychology, 64(4), 742-751.

Blanchard, E. B., Hickling, E. J., Taylor, A. E., \& Loos, W. R. (1994). The psychophysiology of motor vehicle accident related posttraumatic stress disorder. Behavior Therapy, 25(3), 453-467.

Blanchard, E. B., Hickling, E. J., Taylor, A. E., Loos, W. R., Forneris, C. A., \& Jaccard, J. (1996). Who develops PTSD from motor vehicle accidents? Behaviour Research and Therapy, 34(1), 1-10.

Blanchard, E. B., Hickling, E. J., Veazey, C. H., Buckley, T. C., Freidenberg, B. M., Walsh, J. D., et al. (2002). Treatment-related changes in cardiovascular reactivity to trauma cues in motor vehicle accident-related PTSD. Behavior Therapy, 33(3), 417-426.

Blanchard, E. B., Kolb, L. C., Taylor, A. E., \& Wittrock, D. A. (1989). Cardiac response to relevant stimuli as an adjunct in diagnosing post-traumatic stress disorder: Replication and extension. Behavior Therapy, 20(4), 535-543.

Boudewyns, P. A., \& Hyer, L. (1990). Physiological response to combat memories and preliminary treatment outcome in Vietnam veteran PTSD patients treated with direct therapeutic exposure. Behavior Therapy, 21(1), 63-87.

Buckley, T. C., \& Kaloupek, D. G. (2001). A meta-analytic examination of basal cardiovascular activity in posttraumatic stress disorder. Psychosom Med, 63(4), 585-594.

Casada, J. H., Amdur, R., Larsen, R., \& Liberzon, I. (1998). Psychophysiologic responsivity in posttraumatic stress disorder: Generalized hyperresponsiveness versus trauma specificity. Biological Psychiatry, 44(10), 1037-1044.

First, M. B., Spitzer, R. L., Gibbon, M., \& Williams, J. B. W. (1996). Structured clinical interview for DSM-IV, $I V$, patient edition (SCID-I/P)-version 2.0. New York: Biometrics Research Department, New York State Psychiatric Institute.

Foa, E. B., \& Rothbaum, B. (1998). Treating the trauma of rape. New York: Guilford Press.

Hautzinger, M., Bailer, M., Worall, H., \& Keller, F. (1994). Beck-depressions-inventar (BDI). Bearbeitung der deutschen Ausgabe. Testhandbuch. Bern, Göttingen, Toronto, Seattle: Huber.

Hickling, E. J., \& Blanchard, E. B. (1997). The private practice psychologist and manual-based treatments: Posttraumatic stress disorder secondary to motor vehicle accidents. Behaviour Research and Therapy, 35(3), 191-203.

Karl, A., Malta, L. S., Alexander, J., \& Blanchard, E. B. (2004). Startle responses in motor vehicle accident survivors: A pilot study. Applied Psychophysiology and Biofeedback, 29(3), 223-231.

Keane, T. M., Kolb, L. C., Kaloupek, D. G., Orr, S. P., Blanchard, E. B., Thomas, R. G., et al. (1998). Utility of psychophysiological measurement in the diagnosis of posttraumatic stress disorder: Results from a 
Department of Veterans Affairs Cooperative Study. Journal of Consulting and Clinical Psychology, 66(6), 914-923.

Krohne, H. W., Egloff, B., Kohlmann, C. W., \& Tausch, A. (1996). Untersuchungen mit einer deutschen version der "positive and negative affect schedule" (PANAS). Diagnostica, 42, 139-156.

Lang, P. J., Öhmann, A., \& Vaitl, D. (1988). The international affective picture system. Gainesville, Fla.: Univerity of Florida.

Laux, L., Glanzmann, P., Schaffner, P., \& Spielberger, C. D. (1981). Das state-trait-angstinventar. Weinheim: Beltz-Test.

Maercker, A., Zollner, T., Menning, H., Rabe, S., \& Karl, A. (2006). Dresden PTSD treatment study: Randomized controlled trial of motor vehicle accident survivors. BMC Psychiatry, 6, 29.

Marshall, R. D., Olfson, M., Hellman, F., Blanco, C., Guardino, M., \& Struening, E. L. (2001). Comorbidity, impairment, and suicidality in subthreshold PTSD. American Journal of Psychiatry, 158(9), 1467-1473.

McDonagh-Coyle, A., McHugo, G. J., Friedman, M. J., Schnurr, P. P., Zayfert, C., \& Descamps, M. (2001). Psychophysiological reactivity in female sexual abuse survivors. Journal of Traumatic Stress, 14(4), 667683.

Orr, S. P., Lasko, N. B., Metzger, L. J., Berry, N. J., Ahern, C. E., \& Pitman, R. K. (1998). Psychophysiologic assessment of women with posttraumatic stress disorder resulting from childhood sexual abuse. Journal of Consulting and Clinical Psychology, 66(6), 906-913.

Orr, S. P., Lasko, N. B., Shalev, A. Y., \& Pitman, R. K. (1995). Physiologic responses to loud tones in Vietnam veterans with posttraumatic stress disorder. Journal of Abnormal Psychology, 104(1), 75-82.

Orr, S. P., Metzger, L. J., Lasko, N. B., Macklin, M. L., Hu, F. B., Shalev, A. Y., et al. (2003). Physiologic responses to sudden, loud tones in monozygotic twins discordant for combat exposure: Association with posttraumatic stress disorder. Archives of General Psychiatry, 60(3), 283-288.

Orr, S. P., Meyerhoff, J. L., Edwards, J. V., \& Pitman, R. K. (1998). Heart rate and blood pressure resting levels and responses to generic stressors in Vietnam veterans with posttraumatic stress disorder. Journal of Traumatic Stress, 11(1), 155-164.

Rabe, S., Beauducel, B., Zöllner, T., Maercker, A., \& Karl, A. (in press). Regional brain electrical activity in posttraumatic stress disorder after motor vehicle accident. Journal of Abnormal Psychology.

Schnyder, U., \& Moergeli, H. (2002). German version of clinician-administered PTSD scale. Journal of Traumatic Stress, 15, 487-492.

Schuetzwohl, M., \& Maercker, A. (1999). Effects of varying diagnostic criteria for posttraumatic stress disorder are endorsing the concept of partial PTSD. Journal of Traumatic Stress, 12, 155-165.

Shalev, A. Y., Orr, S. P., \& Pitman, R. K. (1992). Psychophysiologic response during script-driven imagery as an outcome measure in posttraumatic stress disorder. Journal of Clinical Psychiatry, 53(9), 324-326.

Shalev, A. Y., Orr, S. P., \& Pitman, R. K. (1993). Psychophysiologic assessment of traumatic imagery in Israeli civilian patients with posttraumatic stress disorder. American Journal of Psychiatry, 150(4), 620-624.

Tomarken, A. J., Davidson, R. J., Wheeler, R. E., \& Kinney, L. (1992). Psychometric properties of resting anterior EEG asymmetry: Temporal stability and internal consistency. Psychophysiology, 29(5), 576-592.

Veazey, C. H., Blanchard, E. B., Hickling, E. J., \& Buckley, T. C. (2004). Physiological responsiveness of motor vehicle accident survivors with chronic posttraumatic stress disorder. Applied Psychophysiology and Biofeedback, 29(1), 51-62.

Watson, D., Clark, L. A., \& Tellegen, A. (1988). Development and validation of brief measures of positive and negative affect: The PANAS scales. Journal of Personality and Social Psychology, 54(6), 1063-1070.

Wittchen, H.-U., Wunderlich, U., Gruschwitz, S., \& Zaudig, M. (1997). SKID. Strukturiertes klinisches interview für DSM-IV. Achse I. Göttingen: Hogrefe.

Zöllner, T., Karl, A., Maercker, A., Hickling, E. J., \& Blanchard, E. B. (2005). Manual zur kognitiven Verhaltenstherapie von Posttraumatischen Belasttungstörungen bei Verkehrsunfallopfern. Lengerich: Pabst Sience Publishers. 